\title{
Enhanced Combustion in Supersonic Flows Using a Pulsed Detonation
}

\author{
Yasin M. Abul-Huda*, and Mirko Gamba ${ }^{\dagger}$, \\ The University of Michigan, Ann Arbor, MI 48109
}

\begin{abstract}
The combustion effects of a hydrogen-oxygen $\left(\mathrm{H}_{2} / \mathrm{O}_{2}\right)$ pulsed detonation (PD) staged downstream of a transverse hydrogen jet in supersonic cross flow (JICF) was investigated. A high-enthalpy $(M=2.35$, $T=1330 \mathrm{~K}, P=40 \mathrm{kPa}$ ) crossflow representative of Mach 7 flight combustor entry conditions was generated and maintained for all test cases. The PD provided a brief, high temperature, high momentum flux ratio, radical-rich, plume of gas to the wake of the jet. The temporal evolution of the blowdown process and interaction between the two plumes was studied using high-speed schlieren and $\mathrm{OH}^{*}$ chemiluminescence imaging. $\mathrm{OH}$ planar laser-induced fluorescence of the center-plane at various times of the blowdown process was used to image the instantaneous reaction zone. The jetto-crossflow momentum flux ratio $\left(J_{j}\right)$ was varied between values of $0.5,2.7$, and 5 to span a range of jet flame stabilization regimes.
\end{abstract}

\section{Introduction}

$\mathrm{T}$ he development of scramjet technology for commercial flight and space access is contingent upon improving the mixing and ignition characteristics within supersonic air-breathing engines. The constrained and dynamic environment present in such combustors poses a number of challenges such as cold start/ignition, mixing, flame stabilization, and complete combustion. Furthermore, the concern for these issues grows as the size of the vehicles are scaled to larger systems. As a result, numerous geometric configurations employing both passive and active methods have been explored to circumvent challenges in both mixing and combustion present in compressible environments with short residence times, low-pressures, and low-temperatures [1,2]. These methods are designed to assist in distributing fuel to the core flow, increase the flow residence time, or provide a region of high energy to the flow to help initiate chemical reactions.

The physical mechanism behind most passive methods relies on the generation of streamwise vorticity, leveraging self-excited resonance, or acoustic excitation. Examples include cavity flame-holders, hypermixers, transverse injection, and shock-shear layer interactions [3-9]. On the other hand, active methods primarily rely on forced excitation of large scales and include methods such as, pulsed jets, piezoelectric actuation, and plasma discharge [10-16]. Each of these methods have been shown to have certain strengths but are ultimately limited by deficiencies such as stagnation pressure losses, combustion confinement near walls, limited range of operation, and significant amounts of input energy required to be effective.

Reacting and non-reacting transverse jets in supersonic crossflow have been studied extensively throughout the past several decades [17-23]. Figure 1 is a three dimensional schematic of such a configuration showing the prominent features that are generated. The underexpanded jet issues into the freestream generating a deflected barrel shock structure which is terminated by a Mach disk. Furthermore, a shear layer is formed between the boundary of the barrel shock and the crossflow. Recirculation regions upstream and downstream of the jet exist due to the separated boundary layer. Several vortical structures are formed during the interaction of the two fluid streams [24] such as: jet shear-layer vortices which form from the Kelvin-Helmholtz instability associated with the annular shear layer of the jet separating from the orifice; horse shoe vortices which wrap around the base of the jet and extend downstream through the wake, a counter-rotating vortex pair (CVP) which results from the aerodynamic forces of the jet onto the crossflow and contributes largely to the entrainment of the crossflow into the wake of the jet; and wake vortices which are intermittent and localized separation events that originate in the boundary layer due to an adverse pressure gradient in the wake. While shear-layer and wake vortices are essentially unsteady in nature, horseshoe and CVP vortices maintain defined mean-flow structures. Furthermore, it is the stream-wise vorticity associated with the CVP that is responsible for most of the far-field mixing, while the other forms of vorticity contribute primarily in the near-field.

The induced flow blockage from the jet generates a bow shock that surrounds the jet boundary. The bow shock can be nearly normal just upstream of the jet, and decays in strength as it wraps around the jet boundary. Moreover,

\footnotetext{
${ }^{*}$ Graduate Research Assistant, Department of Aerospace Engineering, AIAA Student Member, yas inma@umich . edu

${ }^{\dagger}$ Assistant Professor, Department of Aerospace Engineering, AIAA Member, mirkog@umich . edu
} 
the presence of the bow shock creates an adverse pressure gradient in the upstream subsonic boundary layer and under certain conditions, can cause the recirculation region to extend further upstream. The recirculation region increases the flow residence time, allowing for the fuel and air to mix and promoting ignition. However, in a practical system such as a scramjet engine, the extent of jet penetration and the presence of a large recirculation region must be limited because of undesirable effects such as increased stagnation pressure losses and unstart.

The trajectory of a jet can be separated into two components where the first is comprised by the motion carrying it further into the crossflow, and the second results from the entrainment of crossflow fluid. Nevertheless, the mean global characteristic of the jet penetration can be defined in terms of the jet-to-crossflow momentum flux ratio as

$$
J_{j}=\frac{\rho_{j e t} U_{j e t}^{2}}{\rho_{\infty} U_{\infty}^{2}}=\frac{P_{j e t} \gamma_{j e t} M_{j e t}^{2}}{P_{\infty} \gamma_{\infty} M_{\infty}^{2}}
$$

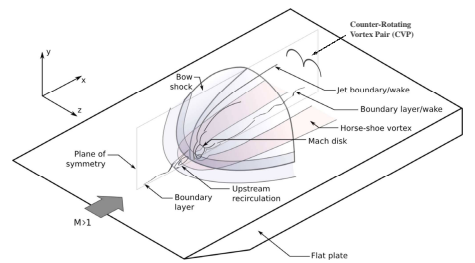

Figure 1. Three dimensional schematic of the canonical transverse jet in supersonic crossflow.

Recently, pulsed detonators (PD's) have been examined as a means of enhancing jet mixing and penetration into the supersonic crossflow by staging a PD downstream of a jet $[25,26]$. The investigations have shown that the global interaction helped distribute and penetrate the upstream jet into the core flow. This results from the large pressure rise across the bow shock of the detonation plume, which acts as a fluidic body that the upstream jet moves around. This also causes the upstream injectant to be carried up into the counter-rotating vortex of the PD plume and deeper into the core flow. In addition, there have been several numerical studies of this configuration aimed to better understand the possible enhancements in ignition and flame stabilization inside of a supersonic combustor. [27,28].

The present study aims to asses the performance of this configuration by experimentally investigating the chemical reaction effects of a staged $\mathrm{H}_{2} / \mathrm{O}_{2}$ PD downstream of a transverse underexpanded sonic hydrogen jet in supersonic crossflow. In particular, we use high-speed schlieren and $\mathrm{OH}^{*}$ chemiluminescence imaging to study the temporal evolution of the interaction of the PD with the primary jet. $\mathrm{OH}^{*}$ imaging was used as a proxy of heat release rate. We specifically document the combustion enhancement that results from the interaction. Furthermore, we use planar laser-induced fluorescence(PLIF) of $\mathrm{OH}$ to mark the distribution of $\mathrm{OH}$ on the center-plane of the flowfield and to infer the instantaneous, local structure of the reaction zones and post-combustion regions as the interaction of the PD with the primary jet evolves. The schlieren movies and OH PLIF images were acquired at the same time.

\section{Experimental Configuration}

\section{A. Expansion Tube Facility and Crossflow Properties}

The high-enthalpy supersonic crossflow used in the present study was generated with the Michigan Hypersonic Expansion Tube Facility (MHExT) [29]. Expansion tubes are capable of accessing a wide range of aerothermal conditions with considerable gains in both stagnation enthalpy and pressure due to the unsteady expansion process used. The operating principles, first introduced by Trimpi [30], rely on processing the test gas through a series of unsteady waves. First, the test gas is compressed by an unsteady shock wave which increases its temperature and pressure as well as induces a bulk velocity. Subsequently, the test gas is expanded to higher Mach numbers through an unsteady expansion wave, which also increases its total enthalpy and pressure. In simplest terms, an expansion tube can be thought of as two shock tubes being operated in tandem, where the first shock tube is comprised of the driver and driven sections, and the second shock tube is comprised of the shock-processed driven section and the expansion section.

The MHExT facility is composed of a $14 \mathrm{~m}$ long stainless steel pipe, comprised of the three main sections. A schematic of the facility is shown in Fig. 2. The driver, driven, and expansion sections have a honed and polished internal diameter of about $144 \mathrm{~mm}$ and are $2.9 \mathrm{~m}, 8 \mathrm{~m}, 3 \mathrm{~m}$ long respectively. The tube is comprised of seven subsections so that test times can be optimized by leveraging its modular design. The entire tube rests on rollers to allow for easy movement of the pipe for diaphragm changing as well as an easier way to interchange the subsections. The dump tank and driver section each rest on their own independent carriage structures. This allows for complete detachment in order to more easily access the interior of the tube for routine inspections and cleaning. 


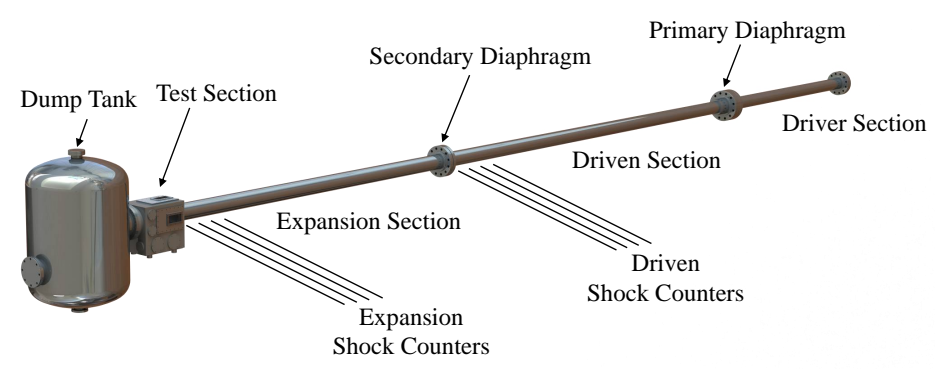

Figure 2. Schematic diagram of the MHExT Facility.

A test section and dump tank are located at the end of the expansion section and are mated with one another through a rubber expansion joint. This allows the dump tank to recoil relative to the test section during each test run, while maintaining the required sealing. The test section is a stainless steel rectangular chamber $45.7 \mathrm{~cm} \times 48.3 \mathrm{~cm}$ $\times 34.3 \mathrm{~cm}$ in size. Three quartz windows are used for optical access through the test section in order to carry out non-intrusive diagnostics. Several feed-through ports and access panels are distributed along its sides. The test section is independently anchored to the laboratory's floor and it is joined to the expansion section through dynamic o-rings to allow for relative movement of the tube while providing vacuum-tight sealing. The dump tank is approximately 1 cubic meter in volume and was designed to be sufficiently large such that properties in the test gas are constant during the usable test time.

Due to the inherently large convective speeds of the wave processes and test gas, data such as those from pressure transducers are sampled at a rate of $2 \mathrm{MHz}$. Data acquisition and control of the system is accomplished using a computer based National Instruments system. The NI system is comprised of a 9-Slot PXIe-1078 Chassis which houses two NI PXI-6133 DAQs, one NI PXI-2521 relay module, and a PXIe-PCIe8381 controller. The system is instrumented with analog and digital input/output modules and a Labview program which is used along with a multitrigger digital delaty generator to control various solenoid valves, MKS Baratron transducers, PCB sensors, lasers, and cameras.

Over seventy side-wall ports and plugs are located along the tube to provide access for instrumentation, such as pressure transducers, and optical access for non-intrusive laser diagnostics measurements. To measure the speed of the primary and secondary shockwaves, eight PCB Piezotronics 113B27 pressure sensors are used. Four of the sensors are mounted into ports spaced $30.5 \mathrm{~cm}$ apart located just upstream of the secondary diaphragm, with a similar set up just upstream of the test section. The shock speeds $U_{s 1}$ and $U_{s 2}$ are computed from the time of flight between sensors, and are then used to determine the test gas conditions using a solver which models expansion tubes and includes equilibrium temperature-dependent properties.

Before operation each section of the tube is evacuated with its own designated vacuum pump. During the fill and evacuation process, the pressures in the driven and expansion sections are monitored using Baratron 627D temperature regulated capacitance manometers. These sensors have a resolution of $20 \mathrm{mtorr}$, and were zeroed and are regularly monitored using thermocouple gauge tubes.

A series of calibration experiments were initially carried out to measure the mean bulk properties of the generated test gas flow. The nominal test gas free stream properties for the present study are $M_{\infty}=2.35, P_{\infty}=40 \mathrm{kPa}$, $T_{\infty}=1330 \mathrm{~K}$, and $U_{\infty}=1660 \mathrm{~m} / \mathrm{s}$. The useful test time was measured to be approximately $600 \mu \mathrm{s}$. Statistical analysis of the shot-to-shot variation of the test gas thermodynamic properties was done by measuring the primary and secondary shock speeds and converting to the corresponding variation in the test gas thermodynamic properties using the expansion tube solver. The primary and secondary shock speeds varied within $1.2 \%$ and $0.9 \%$ of their mean values, respectively. This translates to a shot-to-shot variation of approximately $1.1 \%, 2 \%$, and $1.5 \%$ of the bulk Mach number, pressure, and temperature of the test gas, respectively.

\section{B. Transverse Jet}

The configuration studied here was that of a PD staged downstream of a primary underexpanded sonic jet transverse to a supersonic crossflow. The model used to generate the desired injection scheme is shown as a cross-sectional schematic in Fig. 3. It was comprised of a flat plate with a sharp leading edge that housed the PD $9.6 \mathrm{~mm}$ downstream of the primary jet injector. Gaseous hydrogen was used as fuel for the primary jet and was delivered through a $2 \mathrm{~mm}$ 


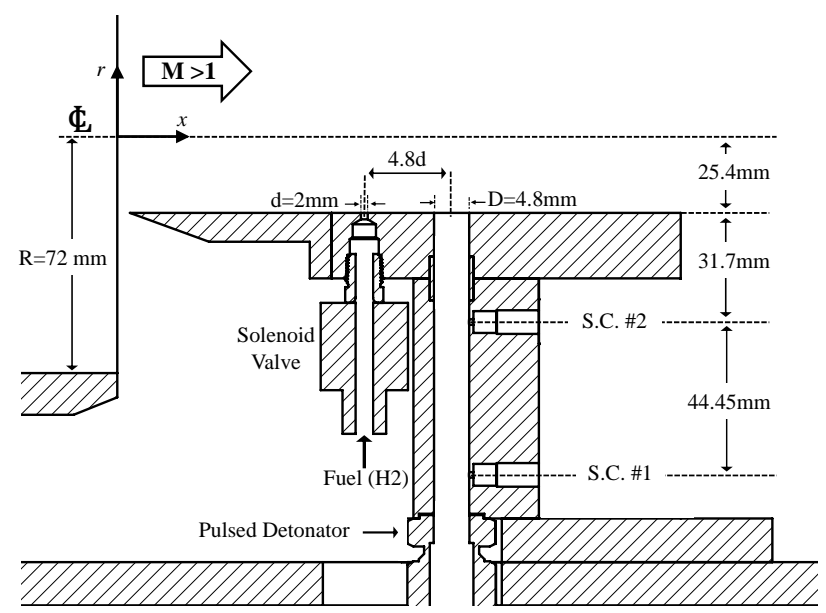

Figure 3. Cross-sectional schematic of experimental test model fixed inside of the MHExT test section.

diameter port. The jet-to-crossflow momentum flux ratio of the primary jet, denoted as $J_{j}$ and given by equation 1 , was varied between values of 0.5, 2.7 and 5. These values were chosen to span a range of flame stabilization regimes [6].

A fast-acting solenoid valve mounted underneath the plate was used to control the fuel injection for the primary jet. Injection was initiated well in advance to ensure a fully-developed and steady jet during the test time. The valve was found to have a response time of approximately $1 \mathrm{~ms}$, and required approximately $3 \mathrm{~ms}$ to achieve a steady and fully developed jet. Calibration of the injection system was carried out to infer the injector (plenum) stagnation pressure as a function of regulator pressure (i.e., upstream of the solenoid valve, which is what we control in the experiment). From this, the mass flow rate and momentum flux through the injector can be evaluated assuming a steady isentropic expansion to choked conditions at the jet exit plane. Calibration was carried out indirectly from measurements of the Mach disk stand-off distance as a function of regulator pressure using known empirical relationships [31,32] between jet stagnation pressure $P_{0 j}$ and Mach disk stand-off distance of underexpanded jets in quiescent media. The estimated stagnation pressure of the primary jets considered in this study is summarized in Table 1.

\section{Pulsed Detonator}

The pulsed detonator was comprised of a $0.75 \mathrm{~m}$ long stainless steel pipe with an inside-diameter of $4.8 \mathrm{~mm}$. Gaseous hydrogen and oxygen were delivered through separate automotive-style fuel injectors at the closed end. The injectors were sized appropriately to completely fill the PD with a stoichiometric mixture, while being opened and closed at the same time. Upon filling the tube, an automotive spark plug located at the closed end of the tube was used to ignite the mixture with approximately $100 \mathrm{~mJ} /$ pulse of energy. Deflagration-to-detonation transition (DDT) was achieved through the use of a $25.4 \mathrm{~cm}$ long schelkin spiral with an area blockage of approximately $50 \%$.

Two piezoelectric (PCB 113B26) sensors spaced $4.45 \mathrm{~cm}$ apart were fixed along the tube $3.17 \mathrm{~cm}$ from the PD exit plane and were used to measure the detonation speed. The acquired signal from the sensors also allowed for temporal synchronization with other devices and identifying the time of arrival of the detonation within the test time. The distribution of the measured detonation speed over 200 shots is shown in Fig. 4a. The mean measured speed was $2800 \mathrm{~m} / \mathrm{s}$ which corresponds to the Chapman-Jouguet (CJ) speed for a stoichiometric hydrogen-oxygen mixture as shown in Fig. 4b. The standard deviation of the measured speed was approximately $40 \mathrm{~m} / \mathrm{s}$. Only experiments with measured detonation speeds within $\pm 3.6 \%$ of $2800 \mathrm{~m} / \mathrm{s}$ were used for analysis since the resulting jump conditions across the detonation, as well as the production of $\mathrm{OH}^{*}$, were predicted to be weakly sensitive to $\phi$ within this range. This is demonstrated in Figs. $4 \mathrm{c}$ and $4 \mathrm{~d}$ where the the pressure and temperature jump, detonation Mach number, and produced mole fraction of $\mathrm{OH}^{*}$ across the $\mathrm{H}_{2} / \mathrm{O}_{2}$ detonation are plotted as a function of equivalence ratio. The grey regions in each figure indicate the predicted range of property variations that were tolerated due to the shot-to-shot variations in the measured detonation speed. The predictions of Fig. 4 were computed using the chemical equilibrium code CEA [33,34]. Therefore, we approximate any variations within this range to have a negligible global effect on the interaction being studied. The expected average temperature and pressure jump ratios across the detonation within the accepted range are then 12 and 18.5, respectively. The extent of jitter, or variability in the time of arrival of the detonation to reach the sensors, was found to be $\pm 15 \mu \mathrm{s}$, which includes the shot-to-shot variation in measured detonation speed as well as the time for DDT to occur. 

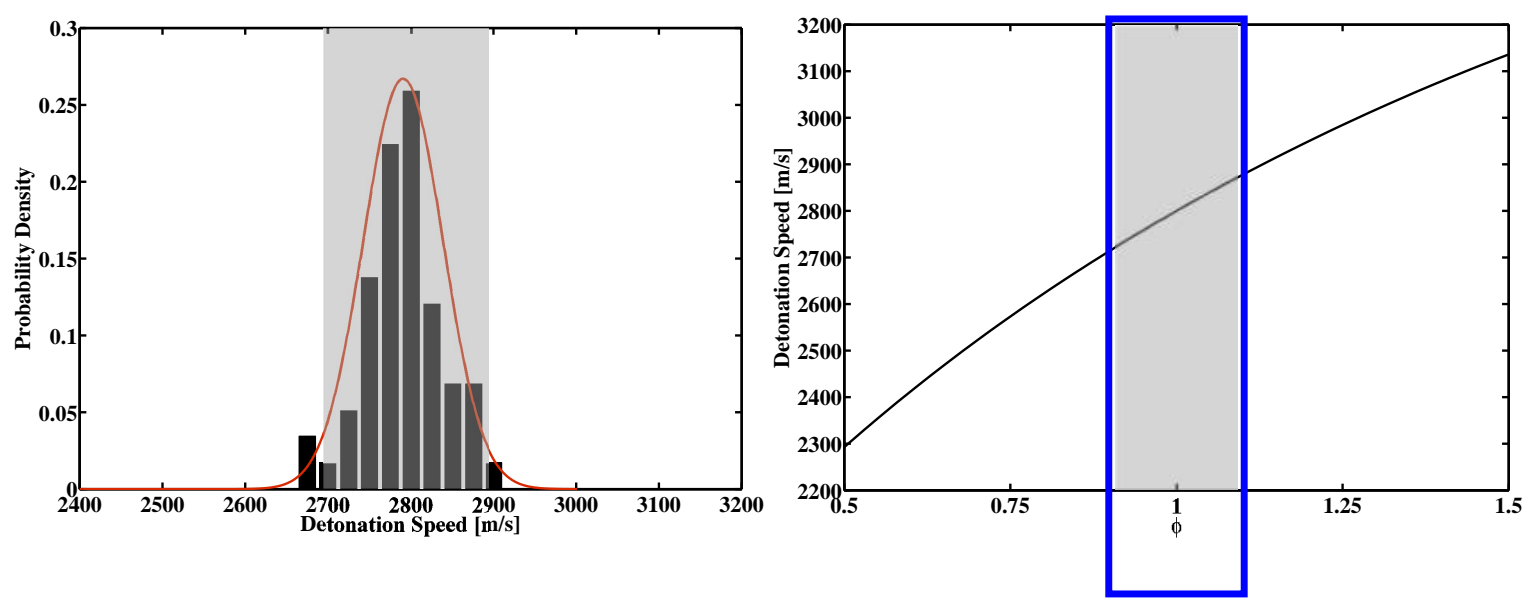

a)

b)

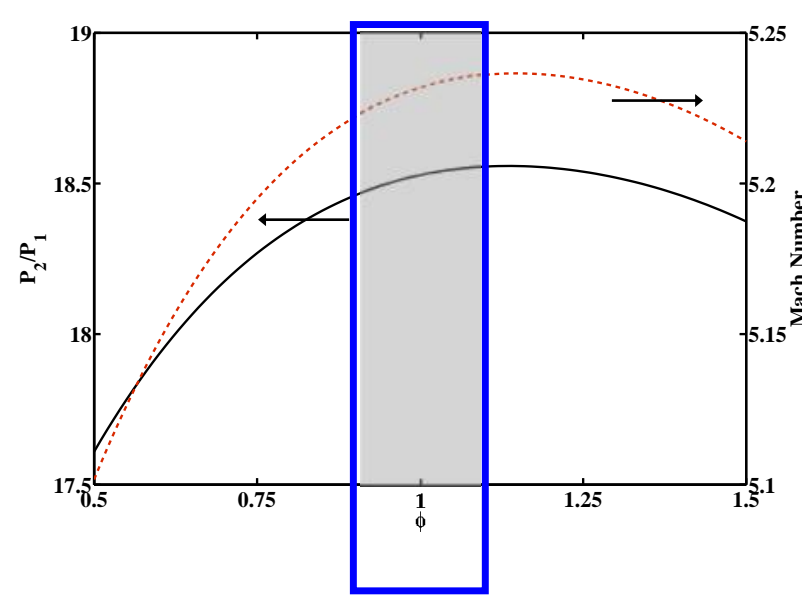

c)

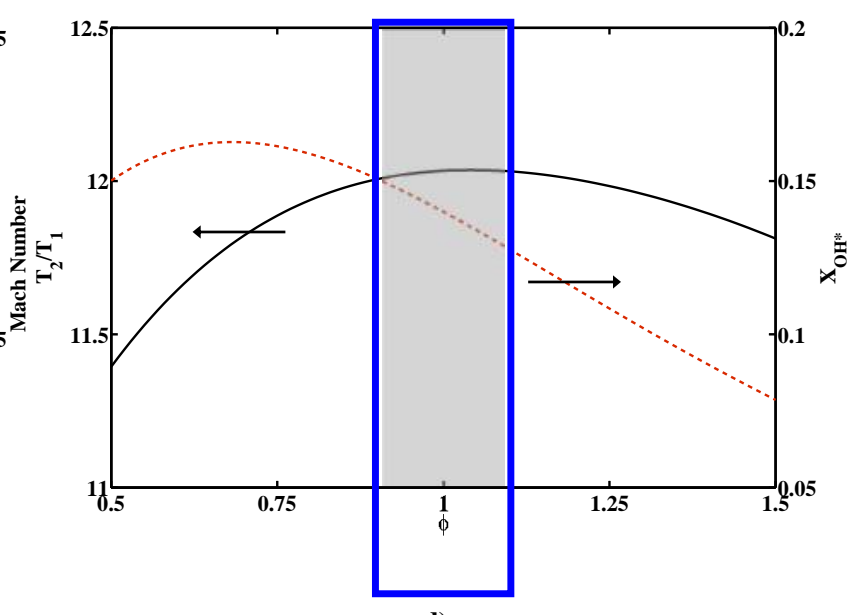

d)

Figure 4. Various properties of a theoretical $H_{2} / O_{2}$ detonation as a function of equivalence ratio for initial conditions ( $P_{1}$ and $\left.T_{1}\right)$ of this study. The grey regions between $0.9 \leq \Phi \leq 1.1$ indicate the range of $\Phi$ in which test data was used and their resulting effects on several aerothermodynamic properties of the detonated gas and wave. (a) Experimentally measured detonation speed distribution over 200 shots where the standard deviation, $\sigma=40 \mathrm{~m} / \mathrm{s}$. (b) Theoretical CJ detonation speed. (c) Theoretical pressure jump and detonation Mach number. (d) Theoretical temperature jump and produced $\mathrm{OH}^{*}$ mole fraction.

The features of the wave processes which control the detonation and the resulting blowdown period are essential in interpreting and assessing the performance of the PD in the current study. A number of studies [35,36] have previously characterized the blowdown process for single-cycle detonation tubes. Figure 5 is a simplified space-time diagram of the wave processes present in the PD upon generating a detonation at the closed end. The detonation wave consumes the stoichiometric mixture initially in state (1) to (2). Because of the closed end of the tube, an expansion wave (Taylor wave) moves along with the detonation in order to satisfy the kinematic constraint at the closed end and bring the detonated gas to rest (i.e. $U_{3}=0$ ). Properties such as pressure in state (2) are difficult to measure directly because of the large convective speeds of the waves and the limited response time of the sensors. Instead, the properties at (2) are computed using the equilibrium code CEA [33] along with the measured detonation speed.

The blowdown period begins when the detonation wave reaches the open end of the tube, where it is then partially transmitted and reflected. The transmitted wave diffracts radially and decreases in strength as it propagates outside of the tube. This is a consequence of the combined effect of the sudden change in area and no longer being sustained by a reactive mixture. Because of the higher pressure at the tube exit compared to the external ambient pressure and since the gas in state (2) is subsonic in the lab reference frame, the reflected wave propagates back into the tube through a non-simple region, further expanding the gas inside of the Taylor wave and in state (3) out of the tube. In the present study, the expanded post-detonation gases exhausting out of the PD are what generate what we refer to as the PD plume. After several wave reflections off the ends of the tube, the pressure inside the PD approaches ambient. Figure 6 is an example of the acquired pressure-time history inside the PD during an experimental run. The transmission of 


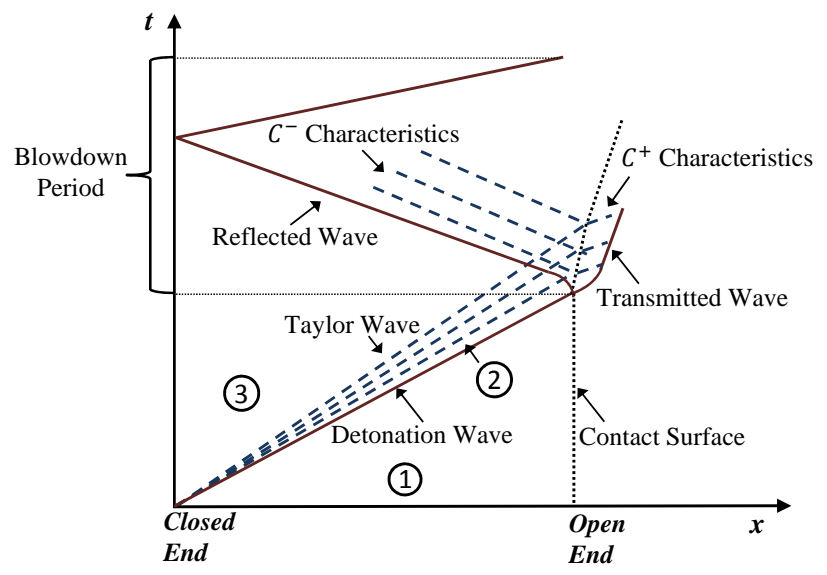

Figure 5. Space-Time diagram of wave processes present during the operation of a pulsed detonator tube.

the detonation wave into the crossflow and the relevant blowdown period where $P \geq P_{\infty}$, lies within the test time which extends from approximately 6.0 to $6.6 \mathrm{~ms}$.

In this study, the PD exhaust process is separated into two components, each with its characteristic value of the momentum flux ratio. The first component, which takes place over several microseconds, is associated with the transmitted wave (von Neumann spike), and its momentum flux ratio $\left(J_{v n}\right)$ is defined in terms of the detonation Mach number and pressure rise (determined from the CEA equilibrium code [14]). The second component is associated with the high-temperature, radical-rich products of the detonation (expanded through the reflected wave), and its characteristic momentum flux ratio $\left(J_{b d}\right)$ is defined assuming that properties evaluated from post-detonation conditions are isentropically expanded to a pressure equal to that measured by the shock counters soon after the detonation wave has passed. A summary of the average momentum flux ratios for the various cases are presented in Table 1 . In the experiments, the value of $J_{b d}$ decreases during the blowdown process (as a result of the decrease in pressure during the blowdown period - see Fig. 6); the tabulated values are the initial values before the pressure in the PD begins to decay. In computing the momentum flux ratio of the plume of the PD we have accounted for the fact that the PD is located in the wake of the primary jet where the pressure is lower than the freestream value [37-39]. Case A is with no upstream injection and only the PD is exhausted into the crossflow. Lastly, it is important to note that for a fixed reacting mixture used in the $\mathrm{PD}$, variations in the PD-to-crossflow momentum flux ratios $\left(J_{v n}\right.$ and $\left.J_{b d}\right)$ can only be achieved by varying the crossflow Mach number and ratio of specific heats. Variations in the crossflow pressure will not alter the values of $J_{v n}$ and $J_{b d}$ significantly because the pressure jump across the detonation wave (see Fig. 4c) is fixed for a given mixture, and therefore so is the ratio $P_{P D} / P_{\infty}$ in Eqn. 1.

It is also important to note in Fig. 6 that the initial pressure inside the PD before the detonation arrives is greater than the crossflow pressure $\left(P / P_{\infty} \approx 1.6\right)$ because the PD is choked during the filling process. This was experimentally verified through separate experiments and the results are summarized in Fig. 7. These results were obtained by mounting a pressure sensor (Kulite EMT-375-500A) in place of the second (downstream) detonation counter to measure the pressure near the open end of the PD. The PD was filled with the same flow rates and hence, reactive mixture used in the study at varying ambient pressures $\left(P_{\infty}\right)$. When using a $4.8 \mathrm{~mm}$ inner diameter pipe, the combined mass flow rates of both automotive fuel injectors was sufficient to choke the PD during the filling stages as long as the ambient pressure was approximately $50 \mathrm{kPa}$ or less. This however, is not the case when a larger inner diameter pipe of $10.3 \mathrm{~mm}$ was used. The pressure near the open end of the PD remains equal to the ambient regardless of how low the ambient pressure is set. Choking of the PD during the filling process is an important requirement in this study because of unsteady wave processes and hence varying pressures, present in the test section before the arrival of the

\begin{tabular}{ccccc}
\hline Case & $J_{j}$ & $P_{0 j}[\mathrm{kPa}]$ & $J_{v n}$ & $J_{b d}$ \\
\hline $\mathbf{A}$ & 0 & 0 & 130 & 2.8 \\
$\mathbf{B}$ & 2.7 & 1095 & 175 & 3.7 \\
$\mathbf{C}$ & 5.0 & 2030 & 260 & 5.6 \\
$\mathbf{D}$ & 0.5 & 200 & 130 & 2.8 \\
\hline
\end{tabular}

Table 1. Test matrix summarizing the average momentum flux ratios for the transverse jet $\left(J_{j}\right)$, and $\operatorname{PD}\left(J_{v n}\right.$ and $\left.J_{b d}\right)$ 


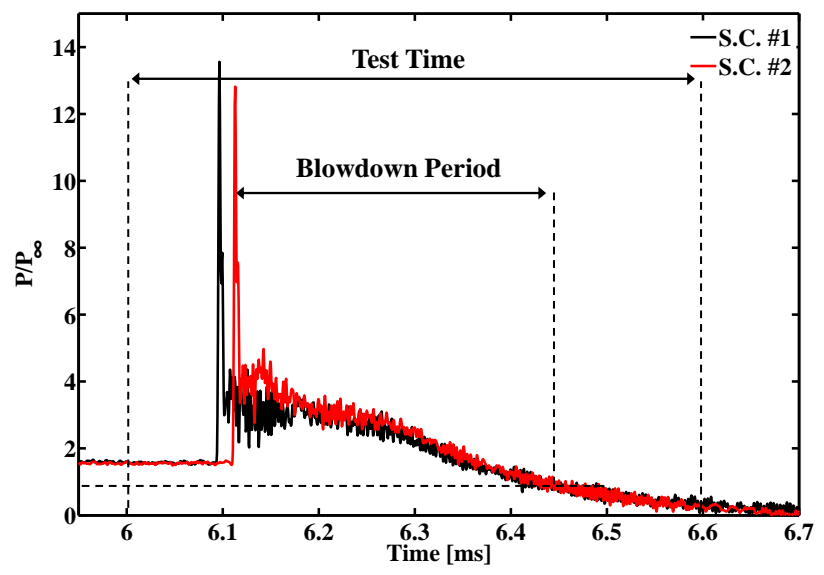

Figure 6. Pressure-time history of a stoichiometric hydrogen-oxygen detonation wave and consequent blowdown process acquired from two piezoelectric pressure sensors mounted in series near the open end of the PD. The data was acquired during an experimental run and the pressure is normalized by the nominal test gas pressure.

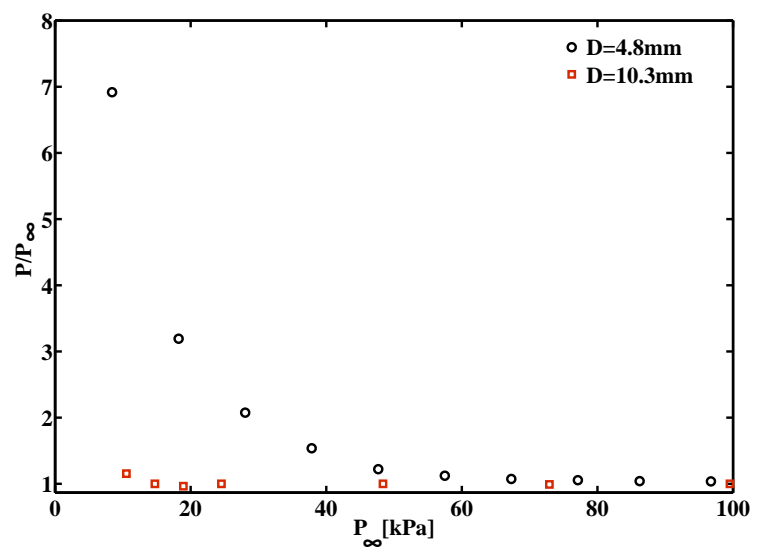

Figure 7. Measured pressure near PD exit during filling process as a function of ambient pressure outside of PD. Circle and square symbols indicate inner pipe diameters of $4.8 \mathrm{~mm}$ and $10.3 \mathrm{~mm}$, respectively.

test gas slug. This is an inherent effect in the operation of the expansion tube facility and is described in more detail in Ref. [29]. Initially, experiments using a $10.3 \mathrm{~mm}$ inner diameter pipe revealed inflow of the shocked expansion gas, which diluted the reactive mixture along the length of the PD. The resulting measured detonation speeds were lower than that of the CJ condition. However, for all cases presented in this work, a $4.8 \mathrm{~mm}$ inner diameter pipe was used for the PD ensuring that it was choked during the filling process. Since the pressure inside the test section never exceeded $50 \mathrm{kPa}$ during the process used to generate the test gas, no inflow was observed to contaminate the PD during the filling process.

\section{Imaging Configuration}

Three imaging techniques were used to study the complex-three dimensional interaction of the staged transverse jet and PD in supersonic crossflow. The temporal evolution of the shock and jet structures was visualized using the schlieren technique. The global characteristics of the chemical reactions were visualized with $\mathrm{OH}^{*}$ chemiluminescence imaging, and the instantaneous reaction zone was visualized with OH PLIF imaging. The schlieren and OH PLIF images were acquired simultaneously.

The schlieren setup consisted of a standard Z-type configuration. The illumination was provided by a continuous LED (Luminus SBR-70), which was collimated/focused with a combination of $2 \mathrm{~m}$ focal length spherical mirrors. A Phantom v771 CMOS camera was used to acquired images at a rate of $63 \mathrm{kHz}$ with an exposure time was $310 \mathrm{~ns}$ which allowed for a temporally frozen flow. White field correction and dark noise subtraction was applied to the recorded images. 
The $\mathrm{OH}^{*}$ chemiluminescence movies were acquired with the same high-speed CMOS camera extended with a high-speed image intensifier (LaVision HS-IRO). Images were taken with a $100 \mathrm{~mm} \mathrm{f} / 2.8 \mathrm{UV}$ lens through a $310 \mathrm{~nm}$ bandpass interference filter $(\mathrm{FWHM}=10 \mathrm{~nm})$ at a rate of $41 \mathrm{kHz}$ with an exposure time of $24.4 \mu$ s to minimize the loss of information between frames, thus fully capturing the transient distribution of heat release.

Single-shot OH PLIF images were acquired with an intensified CCD camera using an optical arrangement similar to what used for the $\mathrm{OH}^{*}$ imaging. Because the OH PLIF images were acquired at the same time with the schlieren movies, the PLIF camera was operated off-axis using a scheimpflug adapter for proper focus on the imaging plane. The excitation laser light source was generated by the frequency-doubled output of an Nd:YAG-pumped tunable dye laser. The $Q_{1}\left(N^{\prime \prime}=7\right)$ line near $283.2 \mathrm{~nm}$ of the $A^{2} \Sigma^{+} \leftarrow X^{2} \Pi\left(v^{\prime}=1, v^{\prime \prime}=0\right)$ electronic transition was used. The acquired images were dewarped to correct for image distortion after background subtraction and laser sheet nonuniformity corrections were applied. However, shot-to-shot variations in energy or spatial non-uniformity were not considered.

The different measurements were acquired in separate runs under nominally identical conditions, and where then used to compile a time-series of the evolution of the PD and primary jet. For the OH PLIF measurements, a series of single-shot images at different time delays from the firing of the PD were acquired to construct a pseudo-time-series of the evolution of the $\mathrm{OH}$ regions of the isolated $\mathrm{PD}$ and of the jet/PD interaction cases.

\section{Interaction of the PD with primary jet}

Here we first describe the impact of the isolated PD blowdown on the crossflow; then, we report our observations on the interaction of the PD and primary jet for various values of $J_{j}$ (see Table 1). A study on the flame structure characteristics of an isolated hydrogen JICF under conditions similar to what is presented here can be found in Ref. [6].

\section{A. Case A: Isolated PD}

Acquired images of the $\mathrm{H}_{2} / \mathrm{O}_{2}$ PD blowdown process into the crossflow without any upstream injection are shown in Fig. 8. Each of the three columns represent a pseudo-time-series of the evolution of the PD visualized by OH PLIF, $\mathrm{OH}^{*}$, and schlieren imaging. The elapsed time since the detonation wave transmits into the crossflow to when each image was acquired is indicated in each figure. Note that the figure was compiled from separate runs and a series of single-shot repetitions. The location of the primary jet and PD is at $x / d=0$ and $x / d=4.8$, respectively. The largest amount of $\mathrm{OH}$ and $\mathrm{OH}^{*}$ are present when the detonation is first transmitted. At later times, the plume penetrates further into the crossflow as it convects through the far-field. The OH PLIF images reveal a continuous distribution of $\mathrm{OH}$ issued from the $\mathrm{PD}$ throughout the entire process. On the other hand, the $\mathrm{OH}^{*}$ images indicate that there is a small presence of the short-lived species after approximately $100 \mu \mathrm{s}$.

The schlieren images reveal the presence of an unsteady shock wave emanating from the PD followed by the formation of a steady bow shock from the large momentum flux ratio $\left(J_{v n}=130\right)$ PD plume until approximately $30 \mu \mathrm{s}$. At $9 \mu \mathrm{s}$ the transmitted detonation wave has left the PD, and transitions to a spherical wave that propagates into the crossflow, while being distorted (see the $25 \mu \mathrm{s}$ frame). At $25 \mu \mathrm{s}$ the spherical wave has penetrated into the crossflow up to $y / d \approx 14$. The crossflow convects the spherical wave downstream, thus distorting it, and leaving behind the high momentum plume of the PD that induces the bow shock. At $57 \mu$ s the value of $J_{b d}$ is approximately 2.8 and decays from that point onward.

It is also interesting to note the presence of $\mathrm{OH}$ and $\mathrm{OH}^{*}$ upstream of the PD exit near the floor at approximately $x / d=1.1$. Their presence correlates well with the position where the the bow shock is attached to the floor. This suggests that a recirculation region upstream of the PD exit, similar to that of a JICF, is generated and where radicals exist with a relatively longer residence time as compared to the bulk mean flow.

\section{B. Case B: $J_{j}=2.7$ With and Without Staged PD}

The effects of the PD staged downstream of a $J_{j}=2.7$ jet are shown in Fig. 9. The left column shows OH PLIF images with and without the PD. The right column shows the corresponding $\mathrm{OH}^{*}$ chemiluminescence images. When no PD is present, the instantaneous shear layer is at approximately $y / d=2.5$ at the jet exit, and grows to approximately $y / d=5$ by the end of the field of view. The reacting shear layer is highly corrugated with an intermittent distribution of $\mathrm{OH}$. There is some evidence of $\mathrm{OH}$ in the wake near the wall, which is a signature of the near-wall burning that is known to occur from the interaction of the JICF with the incoming boundary layer in the near field [6].

With the presence of the PD exhausting in the intitial stages of the blowdown period, both the structure of the reacting shear layer of the primary transverse jet and PD plume is changed. First, the reacting shear layer penetrates deeper into the crossflow, to about $y / d=9$ just downstream of the exit of the PD. The high momentum-flux plume 

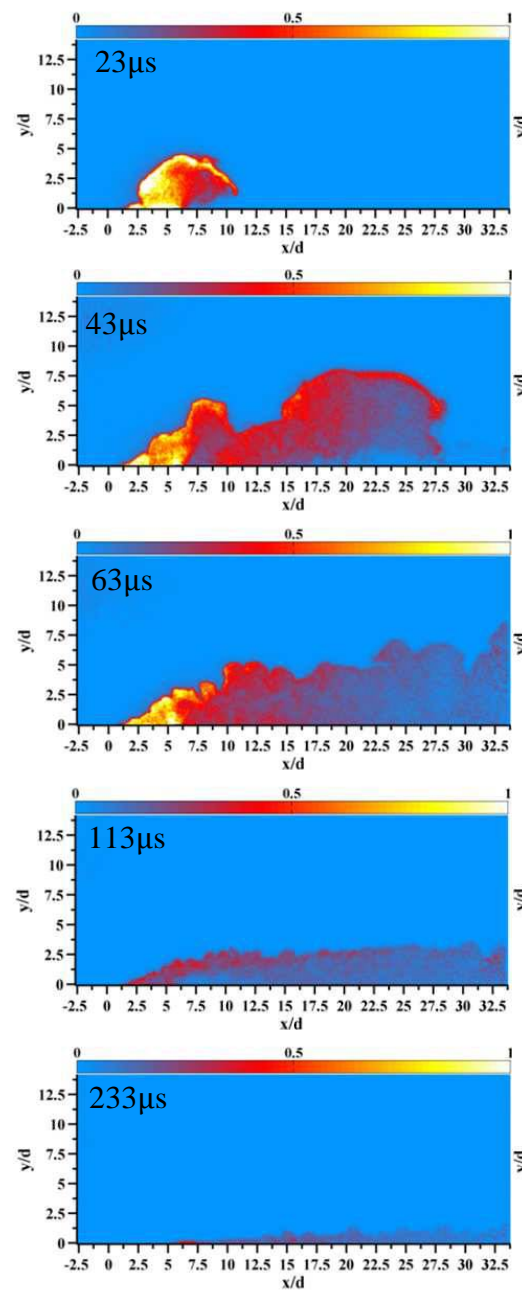

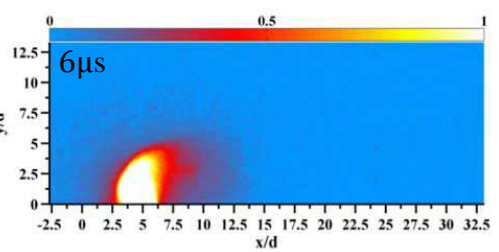

$\mathbf{x} / \mathbf{d}$
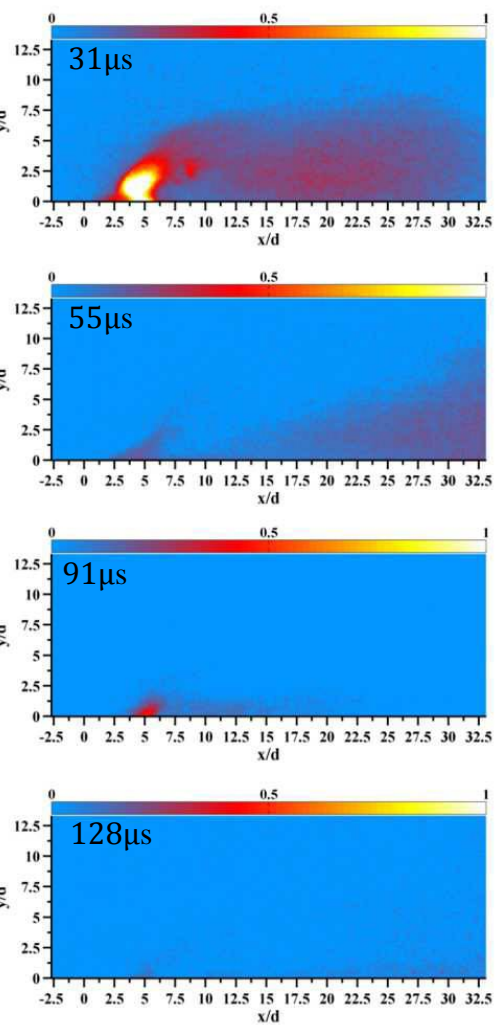

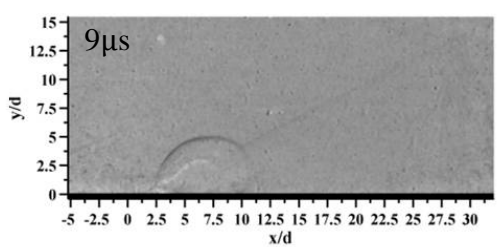

$\mathbf{x} / \mathbf{d}$

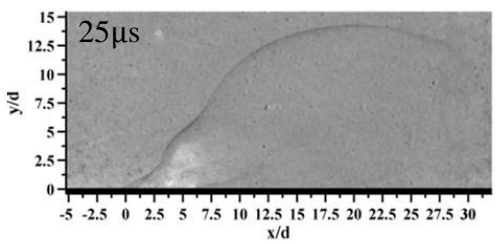

$\mathrm{x} / \mathrm{d}$
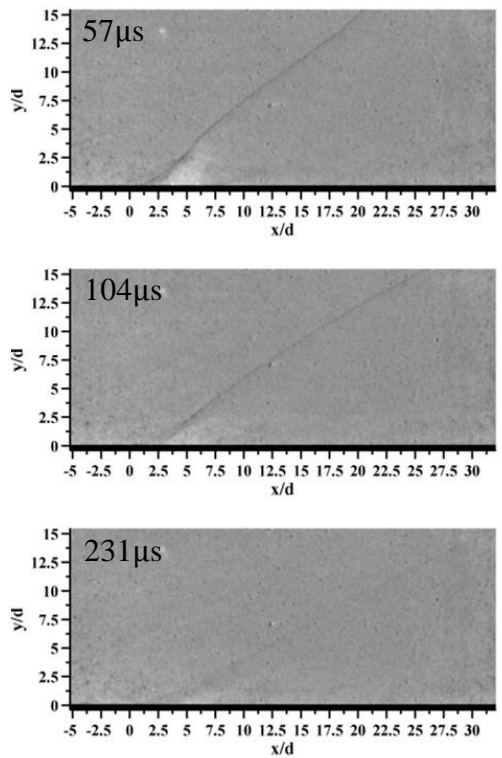

Figure 8. (From left to right) Pseudo-time-series of $\mathbf{O H ~ P L I F , ~} \mathbf{O H}^{*}$, and schlieren images of a $\mathbf{H}_{2} / \mathbf{O}_{2} \mathbf{P D}\left(J_{v n}=130.3\right.$ and $\left.J_{b d}=2.8\right)$ issuing into a Mach 2.35 crossflow.

issued by the PD acts as a fluidic body which displaces the primary jet away from the floor and in turn, generates a stronger bow shock that combines the bow shock formed by the primary jet with that of the PD plume. Second, the shear layer is no longer intermittent, instead, a continuous $\mathrm{OH}$ layer exists as the two plumes interact with one another. Unlike the case of the isolated PD, the presence of the primary jet causes the removal of $\mathrm{OH}$ from the upstream and leeward side of the PD plume. This suggests that a portion of the plume exhausting from the PD may be displaced by the wake of the primary jet.

The $\mathrm{OH}^{*}$ image reveals more insight regarding the interplay of the two plumes. At $43 \mu \mathrm{s}$, there is a large presence of $\mathrm{OH}^{*}$ throughout the flow field, as well as in the vicinity of the PD exit. This is different than the case of the isolated PD (case A). In case A, the $\mathrm{OH}^{*}$ plume from the $\mathrm{PD}$ has decayed significantly throughout the flowfield and especially near the PD exit, when comparing the two cases at an equivalent elapsed time. The plume of $\mathrm{OH}^{*}$, which is used as a relative measure of local heat release, extends to $y / d=8.5$. Moreover, there is a continuous layer of $\mathrm{OH}^{*}$ near the wall and in the far-field downstream of the PD exit, which is not present in the OH PLIF images. This suggests that combustion may be occurring away from the centerplane where the OH PLIF images were taken. It is also interesting to note the similarity of the $\mathrm{OH}^{*}$ plume structure to that of a highly underexpanded jet in supersonic crossflow. In particular, the Mack disk is represented by the intense $\mathrm{OH}^{*}$ region.

\section{Case C: $J_{j}=5.0$ With and Without Staged PD}

At even higher values of $J_{j}$, the characteristics of the jet and PD plume continue to change but retain certain trends and features. The acquired images for a jet momentum flux ratio of 5.0 is shown in Fig. 10. Soon after the detonation wave 

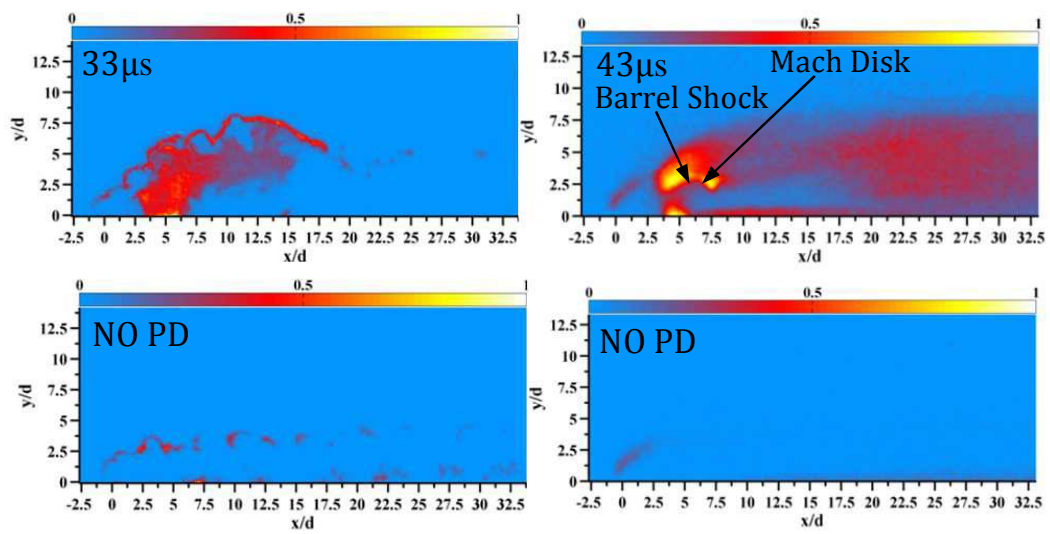

Figure 9. (From left to right) $\mathrm{OH}$ PLIF and $\mathrm{OH}^{*}$ images of a transverse hydrogen jet $\left(J_{j}=2.7\right)$ with a downstream staged $H_{2} / \mathrm{O}_{2}$ PD $\left(J_{v n}=173.7\right.$ and $\left.J_{b d}=3.7\right)$ issuing into a Mach 2.35 crossflow.

is transmitted into the crossflow, it interacts with the wake and the shear layer of the primary jet. A comparison with and without the staged PD reveals an increase of approximately $150 \%$ of the instantaneous reaction zone penetration in the far-field. This is postulated to result from the effectively larger momentum flux ratio of the PD when staged downstream of the jet with a large value of $J_{j}$ because of the low pressure region generated on the leeward side of the JICF $[39,40]$.

The interaction of the primary jet and PD plume causes the shear layer to react more intensely and continuously throughout the measurement region based on the $\mathrm{OH}$ images. We observe a decreased presence of $\mathrm{OH}$ near the wall during the entire blowdown process when compared to the case where no PD was present. We also observe a region of $\mathrm{OH}^{*}$ present deeper in the coreflow (between $52 \mu \mathrm{s}$ and $150 \mathrm{mus}$ ) at the location of the PD exit. At this time, it has not been verified if this is a result of the interaction being studied or if it is a contamination effect in the crossflow generated.

The schlieren images show a similar structure as the transmitted wave is observed to interact and modify the curvature of the bow shock generated by the primary jet. Consequently, the plume of the primary jet is lifted and pushed around the PD plume and further into the crossflow during this high-pressure blowdown period. This unsteady process also causes the jet plume to fluctuate which leads to a dispersion of the jet into the crossflow and PD plume.

Once again we observe that the presence of the upstream JICF causes the effective blowdown time of the PD, where $\mathrm{OH}^{*}$ is present, to extend to longer periods of time.

\section{Case D: $J_{j}=0.5$ With and Without Staged PD}

For case $\mathrm{D}$ when no PD is present the hydrogen being issued from the jet is never ignited within the field of view. The jet remains close to the wall penetrating a distance of approximately $y / d=1$ throughout the field of view. However for case D with a staged PD, the PD plume is observed to carry the upstream primary jet deeper into the cross flow. Measurements of case D reveal a strong similarity to that of case A where the penetration of the reacting shear layer (in the far-field) and transmitted wave are equivalent at the same elapsed times. This is a result of the PD having effectively the same values of momentum flux ratio for the two cases, as is outlined in Table. 1. Staging the PD downstream of a primary transverse jet with a low value of $J_{j}=0.5$ does not increase the penetration of the PD plume, but does increase the penetration of the primary jet as can be seen by the schlieren images at times $24 \mu s$ to 103 $\mu s$. This is in agreement with the $\mathrm{OH}$ images, where a region of $\mathrm{OH}$ is present at the location of the PD exit ( at times between $33 \mu s$ to $65 \mu s$ ). This region of $\mathrm{OH}$ is not present in the isolated PD case. This means that the interaction between the jet and the PD (at the PD exit) generates a larger amount of $\mathrm{OH}$ in the near-field.

The effective blowdown period is longer than that of case A, which reaffirms that the presence of an upstream jet extends the blowdown time over which $\mathrm{OH}^{*}$ is being exhausted from the PD. However, the presence of the primary jet, although with a low value of $J_{j}$, seems to displace the $\mathrm{OH}$ exhausting from the PD near the wall on both the upstream and leeward sides. 

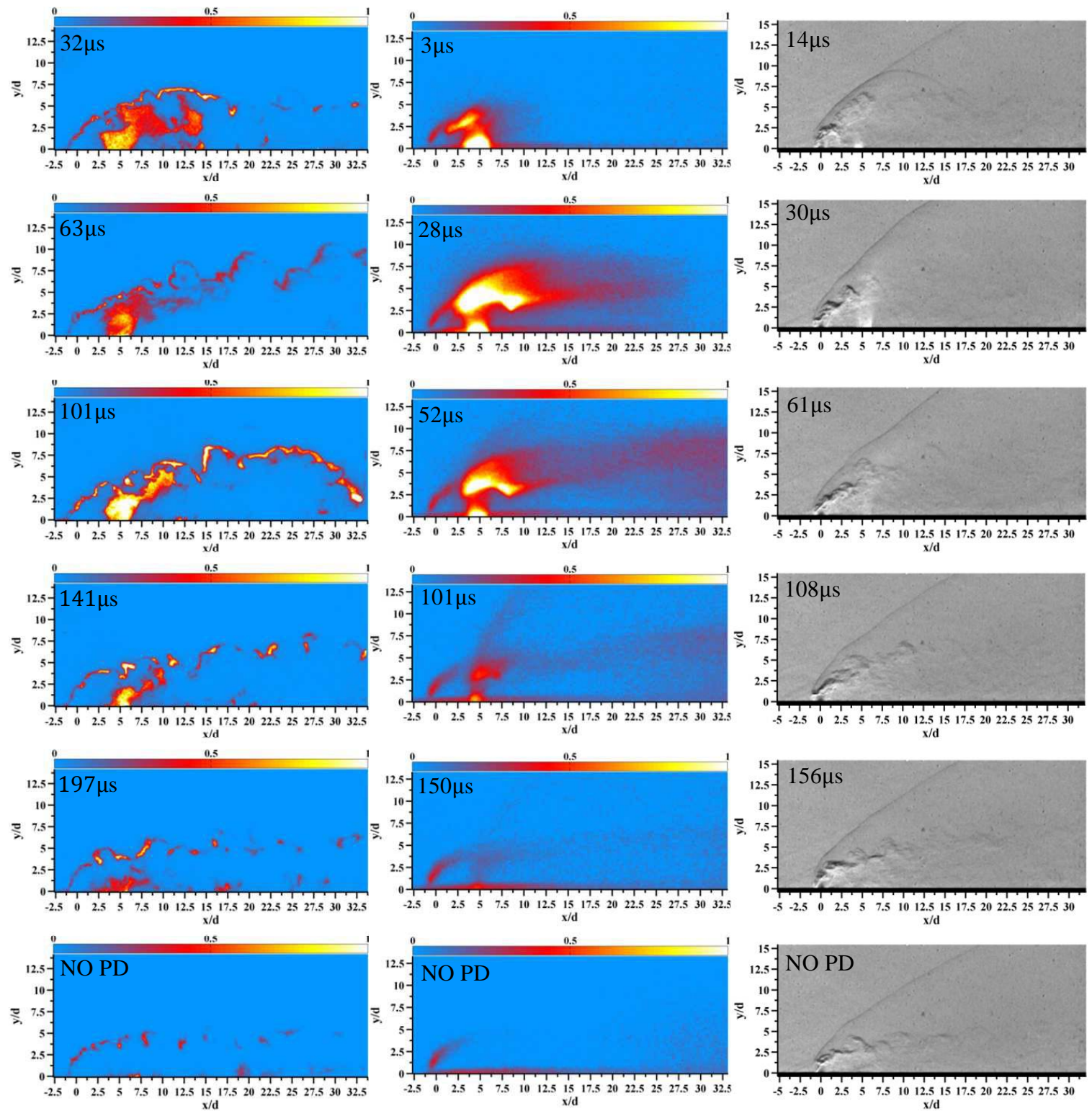

Figure 10. (From left to right) Pseudo-time-series of $\mathrm{OH}$ PLIF, $\mathrm{OH}^{*}$, and schlieren images of a transverse hydrogen jet $\left(J_{j}=5\right)$ with a downstream staged $\mathbf{H}_{2} / \mathbf{O}_{2} \mathbf{P D}\left(J_{v n}=260.5\right.$ and $\left.J_{b d}=5.6\right)$ issuing into a Mach 2.35 crossflow. 

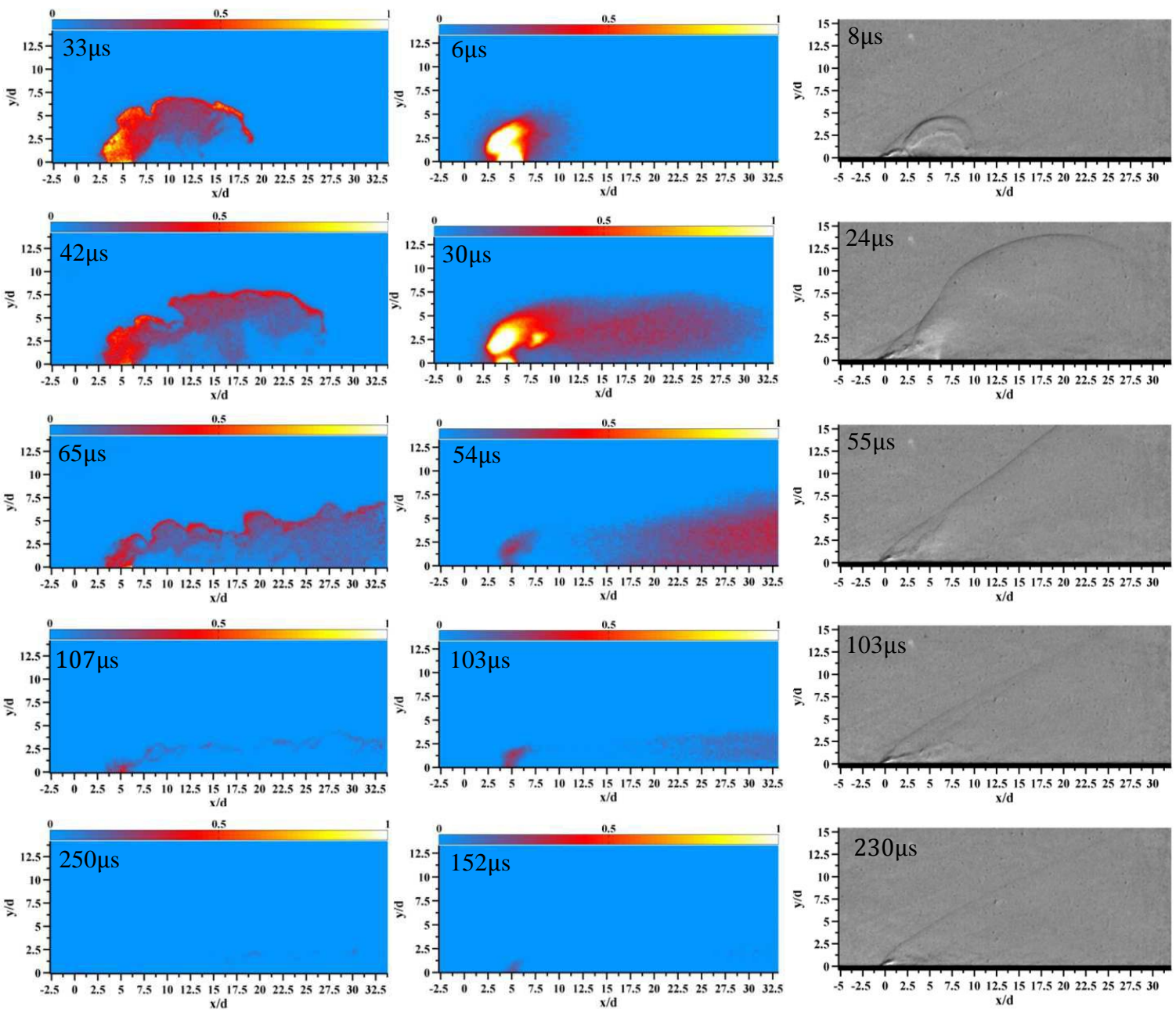

Figure 11. (From left to right) Pseudo-time-series of OH PLIF, OH*, and Schlieren Images of a transverse hydrogen jet $\left(J_{j}=0.5\right)$ with a downstream staged $\mathbf{H}_{2} / \mathbf{O}_{2} \mathbf{P D}\left(J_{v n}=130.3\right.$ and $\left.J_{b d}=2.8\right)$ issuing into a Mach 2.35 crossflow.

\section{E. Comparison Between Cases}

Although the measured $\mathrm{H}_{2} / \mathrm{O}_{2}$ PD blowdown process lasts for approximately $300 \mu \mathrm{s}$ for the configuration used in this study, the effective blowdown period, defined as the time in which $\mathrm{OH}^{*}$ is detected to be exhausting from the PD, lasts for a shorter period of time. This is due to the relatively lower momentum flux ratio present towards the end of the blowdown period, as well as the final portions of the detonation exhaust having been processed by more than one expansion wave inside the PD. Moreover, a dependence of the effective blowdown period on the $J_{j}$ was found, where increasing $J_{j}$ caused an increase in the effective blowdown period.

The penetration of $\mathrm{OH}^{*}$ signal in the far-field from the combined jet and $\mathrm{PD}$ plume into the crossflow was found to scale with values of $J_{j}$, where $y / d=7.5,9.4,13.1$ for the three increasing values of $J_{j}$ investigated. The penetration of the instantaneous reaction zone showed a similar trend, where values of $y / d$ increased by as much as $150 \%$ in the far-field when compared to having no staged PD.

For the cases studied, the interaction of the PD with the wake of the primary jet caused the characteristics of the shear layer to change. Although the instantaneous reacting shear layer remained corrugated, it was significantly less intermittent and remained ignited throughout the flow field. Moreover, with upstream injection the plume of $\mathrm{OH}$ from the PD near the wall seemed to have been displaced for all cases in both the upstream and leeward side of the PD exit. This may be a result of the presence of the CVP of the primary jet which lifts the low momentum exhaust near the wall, along the centerline, into the core flow. This inference is based on the work of Ref. [41], where the velocity field measurements of two staged transverse jets in supersonic crossflow was acquired experimentally.

The penetration of the $\mathrm{OH}$ plume issuing from the $\mathrm{PD}$ at similar time steps of all cases increases further into the crossflow at $x / d \approx 5$ (at the PD exit) as compared to the isolated PD case, even when the upstream jet has a low 
momentum flux ratio of $J_{j}=0.5$. This is an indication that the jet interacts with the PD and causes more $\mathrm{OH}$ to be lifted into the cross flow very early in the interaction. This is supported by the schlieren images of case $\mathrm{D}$ for example, where the jet is observed to be pushed around the PD plume (at the PD exit) between $24 \mu s$ and $103 \mu s$. It may be that the $\mathrm{OH}$ which is no longer present in the leeward side of the jet (just upstream of the PD exit) is being carried into the coreflow by the PD plume.

A simplified schematic summarizing the observations made during this study is illustrated in Fig. 12. The summary combines the observations made from the three imaging techniques used. The schematic of the interaction is at a time after the detonation wave is transmitted and when the PD is exhausting with a mometum-flux ratio of $J_{b d}$ indicated by Table 1. The primary jet and PD are observed to generate deflected barrel shock structures. Each of these structures forms an upstream and a downstream recirculation region. The extent in which $\mathrm{OH}$ was detected within the recirculation regions of the PD port, was affected by the presence (or absence) of the upstream primary jet. With regards to the upstream region, with the isolated PD the $\mathrm{OH}$ signal extended well upstream of the PD exit to nearly $x / d=1.2$. When the upstream primary jet was present, the $\mathrm{OH}$ signal did not extend past $x / d=2.5$. We postulate that this is a displacement effect from the momentum of the upstream primary jet. With regards to the downstream region, the $\mathrm{OH}$ signal did not extend past $x / d=6.2$ when the upstream primary jet was present, whereas it extended through the far-field in the isolated PD case.

The presence of the primary jet forms a bow shock which wraps around both plumes. However, we observe that the strength of the bow shock is altered during the blowdown period of the PD. A steeper bow shock angle was observed between the two ports, as the PD plume penetrated deeper into the coreflow. This is shown in the region labeled (จ). The region labeled (1), just upstream of the PD barrel shock, was found to be rich in $\mathrm{OH}^{*}$ emission. It had a distinct round structure for all cases with upstream injection as compared to the isolated PD. We postulate that it may be as result of the interaction of the impinging primary jet onto the relatively hot and radical-rich PD plume. This region was also always present with $\mathrm{OH}$ only when upstream injection was present. This is not the case with the isolated PD case, indicating an interaction between the primary jet and PD plume.

The region labeled (II) refers to the bright $\mathrm{OH}^{*}$ emission at the location of the PD Mach disk. The flow in this region is subsonic after passing through the shock which seems to enhance chemical reactions. It is also observed in the isolated PD case, however it is much more subtle. Region (III refers to the wake of the combined primary jet and PD plume. The $\mathrm{OH}^{*}$ images reveal significant heat release present within this region during the blowdown period. The amount of time in which this signal is present increases with increasing $J_{j}$.

The reacting shear layer indicated by $\mathbb{N}$ has been observed to have a more continuous distribution of $\mathrm{OH}$ as opposed to the intermittant distribution of the cases without the presence of the staged PD. The boundary of the combined wake of the primary jet and PD has also been shown to penetrate deeper into the core flow with the configuration studied. The large momentum of the PD exhaust is believed to carry the CVP of the primary jet further into the crossflow. This observation is in agreement with the work of Ref. [26], where the transient mixing process of the jet and PD plume was visualized using NO PLIF at several span-wise planes downstream of the PD exit. The large momentum of the PD was observed to initially push the jet outward around the PD plume. Later in the blowdown process, it was found that the jet was entrained within the CVP of the PD plume and carried deeper into the coreflow. Moreover, the study showed that the cross sectional area of the wake of the jet was increased by a factor of six.

We make note of a region of $\mathrm{OH}^{*}$ signal that is present at (NII, for the case when $J_{j}=5.0$. At this point of the study we have not confirmed the cause, or whether or not it is a result of the interaction being studied or particle contaimination in the crossflow itself. Lastly, the region labeled (VII) represents the near-wall burning in the wake of the $\mathrm{PD}$. The emission of $\mathrm{OH}^{*}$ was always greater in this region with the presence of the PD plume. However, $\mathrm{OH}$ seemed to be displaced in this region (along the center plane) when upstream injection was present.

\section{F. Heat Release Analysis}

In this study, the acquired $\mathrm{OH}^{*}$ signal was used as an indicator of the local heat release. Since all acquired $\mathrm{OH}^{*}$ images were taken with the same imaging configuration and settings (intensifier gain, camera lens aperture, etc.), and were processed in an equivalent manner, a relative comparison of heat release distribution was made from the $\mathrm{OH}^{*}$ imaging between cases. An example of the integrated heat release distribution over a portion of the blowdown of Case $\mathrm{C}$ is shown in Fig. 13. Each curve represents a normalized and spatially integrated heat release streamwise distribution at various times, over the exposure time of $24.4 \mu \mathrm{s}$. These curves were generated by vertically integrating the $\mathrm{OH}^{*}$ images of the previous figures. The curves are labeled with increasing integers to indicate later times of the blowdown period. The curve labeled 1 indicates the distribution for the isolated primary transverse jet without the staged PD. For each curve, the location of peak heat release is at the location of the PD exit. After the detonation emerges (curve $2)$, the distributions have two peaks as the PD exhaust decays over time. The first peak $(x / d \approx 5)$ is due to the exhausting $\mathrm{OH}^{*}$ from the $\mathrm{PD}$, and the second $(7 \leq x / d \leq 9)$ is from the $\mathrm{OH}^{*}$ signal that is generated downstream of 


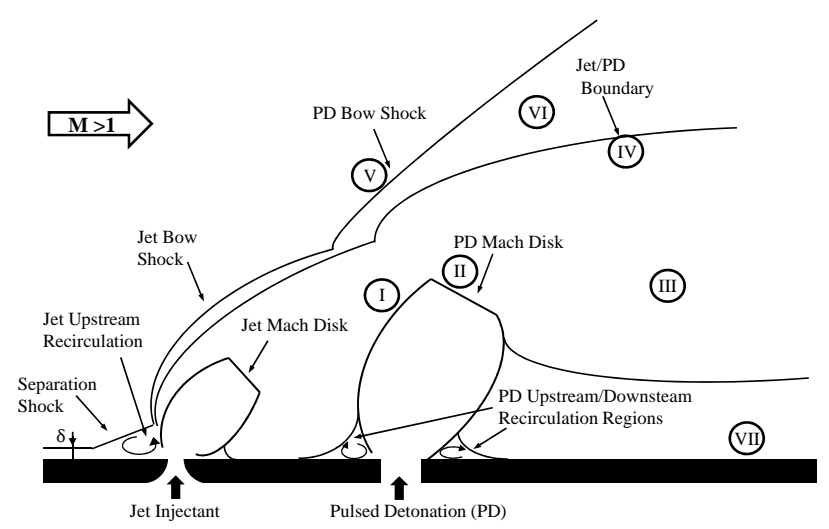

Figure 12. Schematic summary of the observed process present during the interaction of a staged transverse jet and PD in supersonic crossflow. The schematic illustrates the processes at a time soon after the detonation wave has been transmitted.

the Mach disk of the barrel shock (see $\mathrm{OH}^{*}$ images in Fig. 10). As time elapses, the signals from the two peaks decay, however, the presence of $\mathrm{OH}^{*}$ is still higher both upstream of the jet and in the far-field when compared to curve 1. This indicates that there is an increase in heat release within the field of view even at times when the PD exhaust has very low values of $J_{b d}$. Note that the integrated heat release distributions given in Fig. 13 includes contribution from heat release generated from both the primary jet and the PD.

In an attempt to quantify the extent of heat release enhancement caused by the interaction of the PD with the primary jet, the $\mathrm{OH}^{*}$ images of each test case were integrated over a $250 \mu$ s period to determine the total heat release during one PD blowdown cycle. The results for different test cases is shown in Fig. 14a. When no PD is present, the curves indicate increasing distributions of heat release with increasing values of $J_{j}$, with no heat release detected when $J_{j}=0.5$. When a staged PD was present, the extent of heat release increased from the exit of the jet to the far-field. Also shown is the integrated distributed heat release for the isolated PD case. It is interesting to note the more apparent presence of the second $\mathrm{OH}^{*}$ peak $(x / d \approx 8)$ with increasing values of $J_{j}$, whereas with the isolated PD case the second peak is hardly detectable. This is a result of the increasing interplay between the two plumes at increasing values of $J_{j}$.

In order to determine the net effects from the presence of the staged PD on the combustion of the transverse hydrogen jet, the distributed heat release of the isolated PD case was subtracted from those with the transverse jet and staged PD configuration (cases B through D). The results are shown in Fig. 14b. We observe a net increase in heat released with the staged configuration for all values of $J_{j}$ compared to the isolate transverse jet (compare to corresponding curves in Fig. 14a). However, this simplified analysis does not include the effects of the primary jet on the PD. In fact, since the heat released by the PD plume itself may vary depending on the presence of upstream injection, this analysis does not include combustion enhancement effects that the primary jet has on the PD. Only the case where the heat released from the isolated PD was subtracted from the other cases was considered.

The cumulative heat release distribution was computed by performing a cumulative sum $\left(C_{q}\right)$ along the direction of the flow for each test case. The results are shown in Fig. 15. The dash-dot lines show the cumulative heat release for cases without a staged PD. The solid lines are of cases with a staged PD from which the cumulative sum from the case of an isolated PD exhausting into the crossflow has been subtracted. Therefore, the difference in corresponding lines quantifies the amount of enhancement due to the presence of the staged PD. The cumulative heat release is shown to increase by as much as $100 \%$ with the staged PD configuration.

The point of onset of heat release $\left(x_{q}\right)$, defined as the streamwise position at which a significant amount of heat is released, was computed by finding the intercept along the x-axis of the linear fit to $C_{q}(x / d)$ in the portion of the far-field where $C_{q}$ varies linearly. The results are summarized graphically in Fig. 16. In the case where no staged PD is present, the onset of heat release occurs downstream of the jet, and moves further upstream with increasing $J_{j}$. No value of $x_{q} / d$ was computed for the case of $J_{j}=0.5$ because no significant heat release was detected in this case. A similar trend occurs when the staged PD is present. However, the point of onset of heat release when the PD is present is moved well upstream of the jet for large $J_{j}$. Also, the point of onset of heat release for the $J_{j}=0.5$ case was found to be present at approximately the position of the jet. 


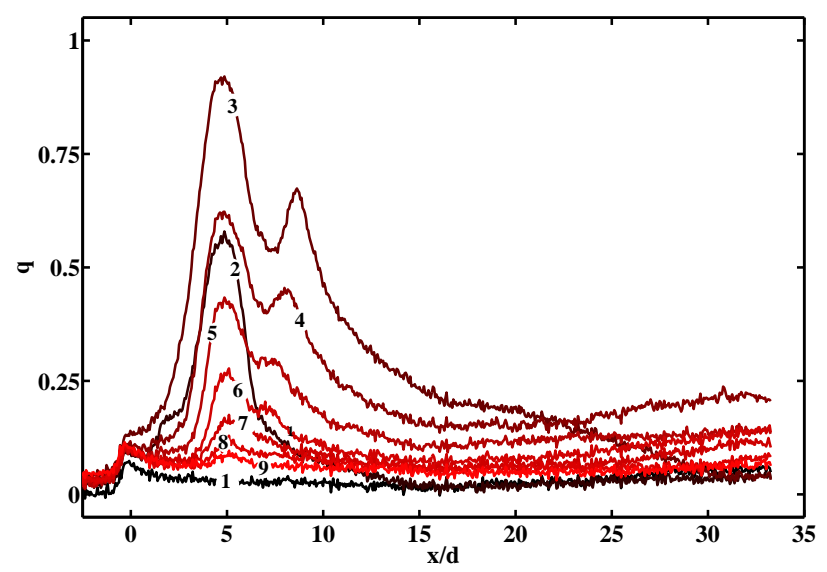

Figure 13. Temporal evolution of normalized and integrated heat release distributions of the blowdown period of case A. Each curve was integrated over a $24.4 \mu s$ exposure. Curves pertaining to later times are labeled with increasing integers.

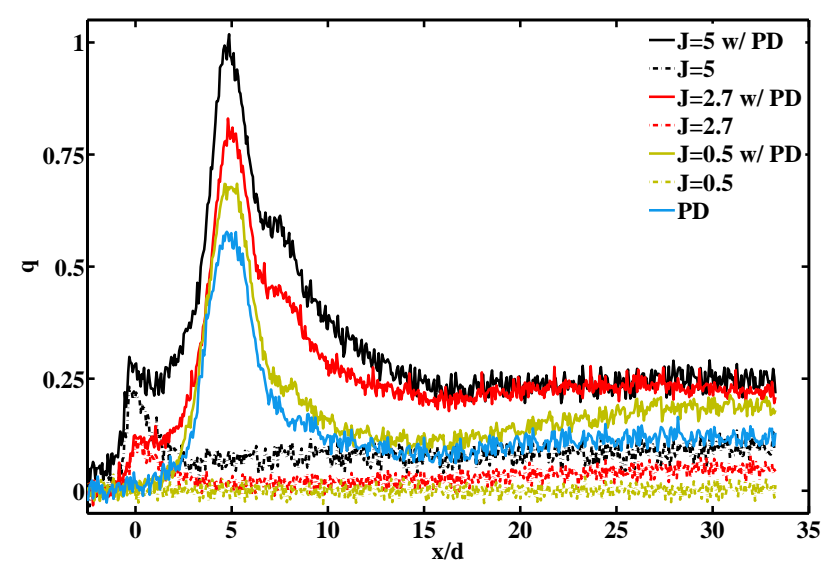

a)

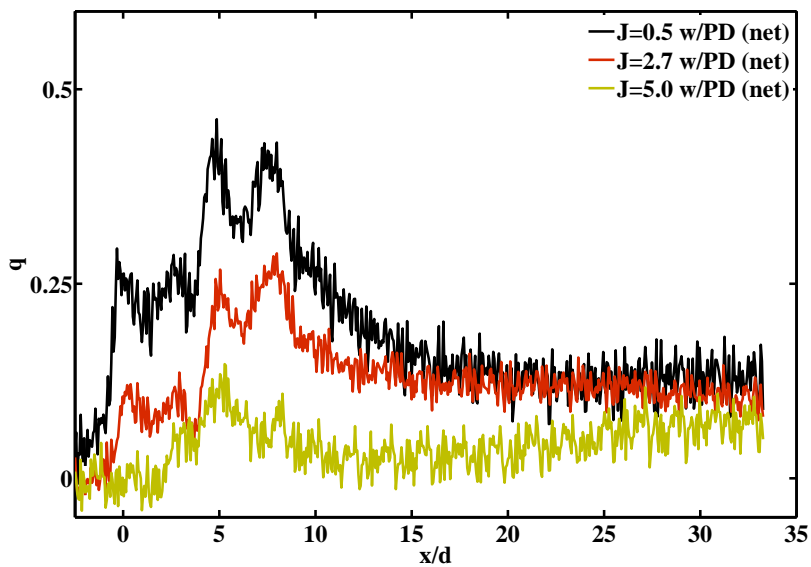

b)

Figure 14. Normalized and integrated heat release distribution over $250 \mu s$ of blowdown period for different test cases. (a) Cases labeled "w/ PD" include heat release from combined presence of primary jet and staged PD. (b) Net gained heat release effects from presence of staged PD.

\section{Conclusions}

The use of a pulsed detonation to enhance the combustion characteristics of a transverse hydrogen jet in supersonic crossflow was experimentally investigated using cinematographic schlieren and $\mathrm{OH}^{*}$ chemilminescence imaging, as well as OH PLIF measurements. The PD provides a high temperature and momentum, radical-rich exhaust to the wake of the transverse jet. The addition of radicals and high temperature gas in the flowfield can alter the combustion progress of the jet, while the addition of the high momentum plume can alter the fluid dynamic structure of the JICF. As a result, the interaction of the two plumes induces several macroscopic changes to the global combustion characteristics of the transverse jet during the PD blowdown process. Some of these macroscopic changes are quantified in this study. In a practical system, the use of a PD as an active method of assisting combustion may be made compact and efficient as it can be operated from the chemical energy already stored on-board an aircraft.

It was found that the instantaneous reaction zone was continuously ignited throughout the field of view when the PD was present, while it was highly intermittent when the PD was not operated. The high-speed schlieren images showed that the interaction of the plumes caused an enhancement in the dispersion and penetration of the jet. Moreover, the high-speed $\mathrm{OH}^{*}$ images indicate that the increased penetration of fuel into the core flow results in a greater penetration of the reacting shear layer and in a larger overall release of heat. The penetration of the shear layer always increased with the presence of the PD; for example for the case with $J_{j}=5$, a $150 \%$ increase in far-field penetration was observed. 


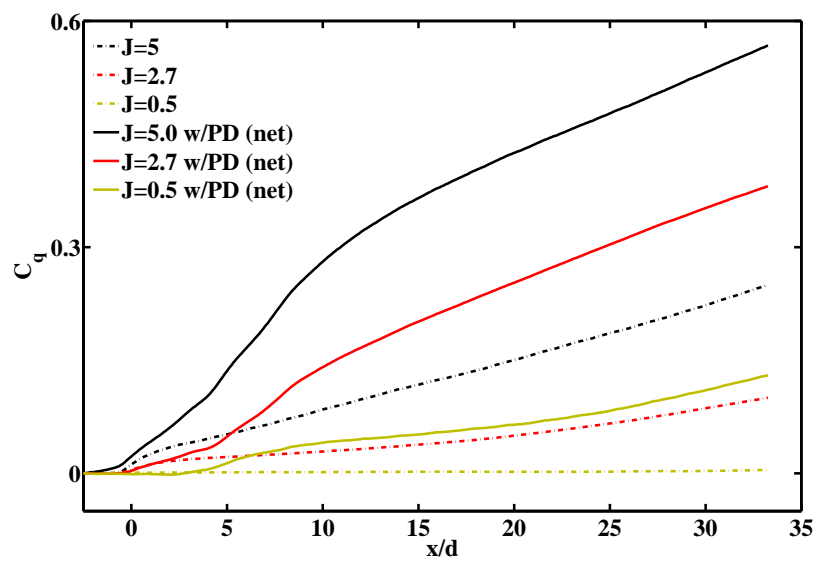

Figure 15. Normalized cumulative heat release for various test cases.

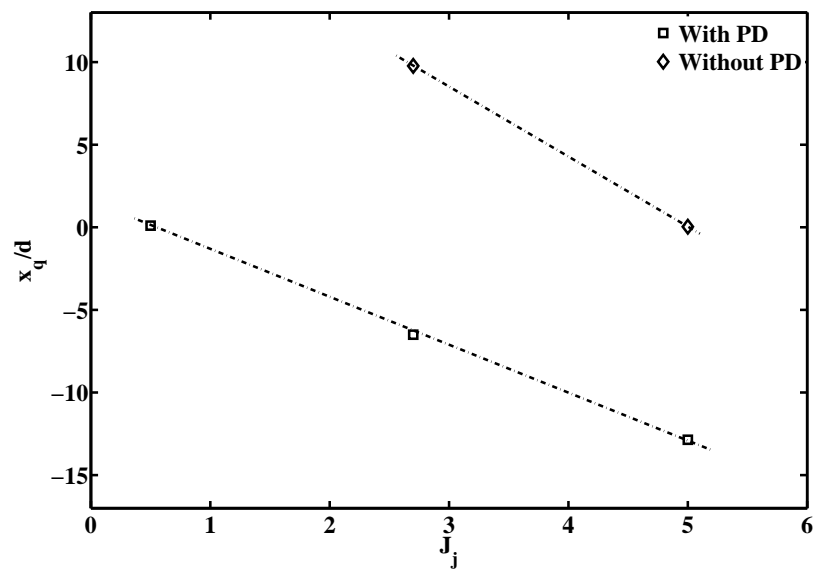

Figure 16. Location of the onset of heat release for various test cases.

The distribution of a relative measure of heat release was quantified using the $\mathrm{OH}^{*}$ chemiluminescence images. With a staged PD, the interaction of the plumes was found to promote ignition and initiate the release of heat closer to the jet. Not only did the PD enhance the combustion characteristics of the primary jet, but it was also found that the interaction of the two plumes changes the evolution of the PD blowdown process itself. For example, the effective blowdown time over which $\mathrm{OH}^{*}$ was exhausted from the PD increases as the momentum flux ratio of the primary jet increases.

\section{Acknowledgments}

Yasin Abul-Huda acknowledges the support of the National Defense Science and Engineering (NDSEG) Fellowship as well as the Rackham Merit Fellowship (RMF).

\section{References}

[1] Seiner, J. M., "Historical Survey on Enhanced Mixing in Scramjet Engines," Journal of Physics D: Applied Physics, Vol. 17, No. 6, 2001

-[2] Gutmark, E. J., Schadow, K. C., and Yu, K., "Mixing Enhancement in Supersonic Free Shear Flows," Annual Review Fluid Mechanics, Vol. 27, 1995, pp. 375-417.

[3] Hsu, K.-Y., Carter, C. D., Gruber, M. R., Barhorst, T., and Smith, S., "Experimental Study of Cavity-Strut Combustion in Supersonic Flow," Journal of Propulsion and Power, Vol. 26, No. 6, Nov. 2010, pp. 1237-1246.

[4] Yu, K. H., Ken, W., and Klaus, S., "Effect of Flame-Holding Cavities on Supersonic-Combustion Performance," Journal of Propulsion and Power, Vol. 17, No. 6, 2001. 
[5] Stouffer, S. D., Baker, N. R., and Capriotti, D. P., "Effects of Compression and Expansion-Ramp Fuel Injector Configurations on Scramjet Combustion and Heat Transfer," 31st Aerospace Sciences Meeting AIAA, 1993.

- [6] Gamba, M. and Mungal, M. G., "Ignition, flame structure and near-wall burning in transverse hydrogen jets in supersonic crossflow," Journal of Fluid Mechanics, Vol. 780, 2015, pp. 226-273.

[7] Ben-yakar, A. and Hanson, R. K., "Cavity Flame-Holders for Ignition and Flame Stabilization in Scramjets : An Overview," Vol. 17, No. 4, 2001.

[8] Vergine, F., Crisanti, M., Maddalena, L., Miller, V., and Gamba, M., "Supersonic Combustion of Pylon-Injected Hydrogen in High-Enthalpy Flow with Imposed Vortex Dynamics," Journal of Propulsion and Power, Vol. 31, No. 1, Jan. 2015, pp. 89103.

-[9] Waitz, A., Marblet, F. E., and Zukoskij, E. E., "Investigation of a Contoured Wall Injector for Hypervelocity Mixing Augmentation,” AIAA Journal, Vol. 31, No. 6, 1993, pp. 1014-1021.

-[10] Do, H., Cappelli, M. a., and Mungal, M. G., "Plasma assisted cavity flame ignition in supersonic flows," Combustion and Flame, Vol. 157, No. 9, Sept. 2010, pp. 1783-1794.

-[11] Do, H., Im, S.-k., Cappelli, M. a., and Mungal, M. G., "Plasma assisted flame ignition of supersonic flows over a flat wall," Combustion and Flame, Vol. 157, No. 12, Dec. 2010, pp. 2298-2305.

[12] Leonov, S. B., Yarantsev, D. A., Napartovich, A. P., and Kochetov, I. V., "Plasma-Assisted Combustion of Gaseous Fuel in Supersonic Duct," IEEE Transactions on Plasma Science, Vol. 34, No. 6, 2006, pp. 2514-2525.

[13] J.Lepicovsky, K.K.Ahuja, W.H.Brown, M.Salikuddin, and P.J.Morris, "Acoustically Excited Heated Jets I-Internal Excitation,” NASA Contractor Report 4129-PT-1, 1988.

[14] Bogdanoff, D. W., “Advanced Injection and Mixing Techniques for Scramjet Combustors,” Jo, Vol. 10, No. 2, 1994, pp. 183190.

- [15] Ombrello, T., Qin, X., Ju, Y., and Carter, C., "Combustion Enhancement via Stabilized Piecewise Nonequilibrium Gliding Arc Plasma Discharge," AIAA Journal, Vol. 44, No. 1, 2006.

- [16] Panton, R. L., "Excitation by Grazing Flow," AIAA Journal, Vol. 28, No. 1, 1989, pp. 60-65.

[17] Rodgers, C., “A Study of the Mixing of Hydrogen Injected Normal to a Supersonic Airstream,” Tech. Rep. March, Langley Research Center, Hampton, Va 23365, 1971.

[18] Rodgers, C., "Mixing of Hydrogen Injected From Multiple Injectors Normal to a Supersonic Airstream,” Tech. Rep. September, Langley Research Center, Hampton, Va 23365, 1971.

[19] Mcmillin, B. K., Seitzman, J. M., and Hanson, R. K., "Comparison of NO and OH Planar Fluorescence Temperature Measurements in Scramjet Model Flowfields," AIAA Journal, Vol. 32, No. 10, 1994.

-[20] Allen, M. G., Parker, T. E., Reinecke, W. G., Legner, H. H., Foutter, R. R., Rawlins, W. T., and Davis, S. J., "Fluorescence Imaging of OH and NO in a Model Supersonic Combustor," AIAA Journal, Vol. 31, No. 3, 1993.

[21] Gruber, M. R., Nejadt, A. S., and Chen, T. H., "Mixing and Penetration Studies of Sonic Jets in a Mach 2 Freestream,” Journal of Prop, Vol. 11, No. 2, 1995.

-[22] Lee, M. P., Mcmillin, B. K., Palmer, J. L., and Hansont, R. K., "Planar Fluorescence Imaging of a Transverse Jet in a Supersonic Crossflow," Journal of Propulsion and Power, Vol. 8, No. 4, 1992.

[23] Schetz, J. A., Hawkins, P. F., and Lehmanf, H., "Structure of Highly Underexpanded Transverse Jets in a Supersonic Stream," AIAA Journal, Vol. 5, No. 5, 1966, pp. 882-884.

-[24] Roshko, A., "Vortical structure in the wake of a transverse jet," Journal of Fluid Mechanics, Vol. 279, 1994, pp. 1-47.

- [25] Ombrello, T., Carter, C., McCall, J., Schauer, F., Naples, A., Hoke, J., and Hsu, K.-Y., "Enhanced Mixing in Supersonic Flow Using a Pulse Detonator," Journal of Propulsion and Power, Vol. 31, No. 2, March 2015, pp. 654-663.

-[26] Ombrello, T., Tam, C.-j., Haw, W., and Carter, C., "Transient Mixing Enhancement of a Transverse Jet in Supersonic Cross Flow Using Pulse Detonation,” Vol. 127, 2015, pp. 247-264.

-[27] Malo-molina, F. J., "Three Dimensional Analysis of a Single Hydrocarbon-Air Pulse Detonation in a Supersonic Combustor," AIAA, 52nd Aerospace Sciences Meeting, No. January, 2014, pp. 1-13.

-[28] Malo-molina, F. J. and Dreyer, E., “Analysis of a Single H 2 -Air Pulse Detonation in a Supersonic Combustor," AIAA, 51st Aerospace Sciences Meeting including the New Horizons Forum and Aerospace Exposition, No. January, 2013.

-[29] Abul-Huda, Y. and Gamba, M., "Design and Characterization of the Michigan Hypersonic Expansion Tube Facility (MHExT),”, No. January, 2015, pp. 1-13.

[30] Trimpi, R., "Preliminary Theoretical Study of the Expansion Tube, A New Device for Producing High Enthalpy Short Duration Hypersonic Gas Flows," NASA Technical Report, 1962.

[31] Ashkenas, H. and Sherman, F., "Structure and Utilization of Supersonic Free Jets in Low Density Wind Tunnels," NASA CR-60423, Vol. 222, No. 45, 1962. 
[32] Crist, S., Sherman, P. M., and Glass, D. R., "Study of the Highly Underexpanded Sonic Jet," AIAA Journal, Vol. 4, No. 1, 1966.

[33] "Chemical Equilibrium with Applications, NASA Glenn Research Center at http://www.grc.nasa.gov/WWW/CEAWeb/" .

[34] Gordon, S. and McBride, B., "Computer Program for Calculation of Complex Chemical Equilibrium Compositions and Applications (NASA-RP1311),” Tech. rep., CEA1, 1994.

[35] Wintenberger, E., Austin, J. M., Cooper, M., Jackson, S., and Shepherd, J. E., "Analytical Model for the Impulse of SingleCycle Pulse Detonation Tube," Journal of Propulsion and Power, Vol. 19, No. 1, 2003.

[36] Endo, T., Kasahara, J., Matsuo, A., Sato, S., Inaba, K., and Fujiwara, T., "Pressure History at the Thrust Walls of a Simplified Detonation Engine,” AIAA Journal, Vol. 42, No. 9, 2004.

-[37] Crafton, J., Forlines, A., Palluconi, S., and Hsu, K.-y., "Investigation of Transverse Jet Injections in a Supersonic Crossflow Using Fast Responding Pressure-Sensitive Paint," AIAA, 29th Applied Aerodyanmics Conference, No. June, crafton2011, 2011.

- [38] Snyder, J. A. and Chang, L. S., "Pressure-Sensitive Paint Measurements of Sonic Jet Injection into Supersonic Crossflow," AIAA 43rd Joint Propulsion Conference and Exhibit, No. July, 2007, pp. 1-7.

-[39] Gruber, M. R. and Goss, L. P., “Surface Pressure Measurements in Supersonic Transverse Injection Flow elds," Journal of Propulsion and Power, Vol. 15, No. 5, 1999.

- [40] Everett, D. E., "Wall Pressure Measurements for a Sonic Jet Injected Transversely into a Supersonic Crossflow," Journal of Propulsion and Power, Vol. 14, No. 6, 1998.

[41] Hollo, S. D., Mcdaniel, J. C., and Jr, R. J. H., "Quantitative Investigation of Compressible Mixing : Staged Transverse Injection into Mach 2 Flow,” AIAA Journal, Vol. 32, No. 3, 1994, pp. 528-534. 